\title{
Colouring random geometric graphs
}

\author{
C. J. H. McDiarmid ${ }^{1}$ and T. Müller ${ }^{1 \dagger}$ \\ ${ }^{1}$ University of Oxford, Department of Statistics, 1 South Parks Road, Oxford OX1 3TG, United Kingdom
}

A random geometric graph $G_{n}$ is obtained as follows. We take $X_{1}, X_{2}, \ldots, X_{n} \in \mathbb{R}^{d}$ at random (i.i.d. according to some probability distribution $\nu$ on $\mathbb{R}^{d}$ ). For $i \neq j$ we join $X_{i}$ and $X_{j}$ by an edge if $\left\|X_{i}-X_{j}\right\|<r(n)$. We study the properties of the chromatic number $\chi_{n}$ and clique number $\omega_{n}$ of this graph as $n$ becomes large, where we assume that $r(n) \rightarrow 0$. We allow any choice $\nu$ that has a bounded density function and $\|\cdot\|$ may be any norm on $\mathbb{R}^{d}$. Depending on the choice of $r(n)$, qualitatively different types of behaviour can be observed. We distinguish three main cases, in terms of the key quantity $n r^{d}$ (which is a measure of the average degree). If $r(n)$ is such that $\frac{n r^{d}}{\ln n} \rightarrow 0$ as $n \rightarrow \infty$ then $\frac{\chi_{n}}{\omega_{n}} \rightarrow 1$ almost surely. If $\frac{n r^{d}}{\ln n} \rightarrow \infty$ then $\frac{\chi_{n}}{\omega_{n}} \rightarrow \frac{1}{\delta}$ almost surely, where $\delta$ is the (translational) packing density of the unit ball $B:=\left\{x \in \mathbb{R}^{d}:\|x\|<1\right\}$ (i.e. $\delta$ is the proportion of $d$-space that can be filled with disjoint translates of $B)$. If $\frac{n r^{d}}{\ln n} \rightarrow t \in(0, \infty)$ then $\frac{\chi_{n}}{\omega_{n}}$ tends almost surely to a constant that can be bounded in terms of $\delta$ and $t$. These results extend earlier work of McDiarmid and Penrose. The proofs in fact yield separate expressions for $\chi_{n}$ and $\omega_{n}$.

We are also able to prove a conjecture by Penrose. This states that when $\frac{n r^{d}}{\ln n} \rightarrow 0$ then the clique number becomes focussed on two adjacent integers, meaning that there exists a sequence $k(n)$ such that $\mathbb{P}\left(\omega_{n} \in\{k(n), k(n)+1\}\right) \rightarrow$ 1 as $n \rightarrow \infty$. The analogous result holds for the chromatic number (and for the maximum degree, as was already shown by Penrose in the uniform case).

Keywords: random geometric graphs, graph colouring

\section{Introduction}

A random geometric graph $G_{n}$ is obtained as follows. We pick vertices $X_{1}, \ldots, X_{n} \in \mathbb{R}^{d}$ at random (iid according to some probability distribution $\nu$ on $\left.\mathbb{R}^{d}\right)$ and we join $X_{i}, X_{j}(i \neq j)$ by an edge if $\left\|X_{i}-X_{j}\right\|<r(n)$, where we assume that $r(n) \rightarrow 0$ as $n \rightarrow \infty$. We will allow any choice of $\nu$ that has a bounded density and $\|\cdot\|$ may be any norm on $\mathbb{R}^{d}$. In this paper we are interested in the behaviour of the clique number, $\omega_{n}$, and the chromatic number, $\chi_{n}$, of $G_{n}$ as $n$ grows large. The distance $r(n)$ plays a role similar to that of $p(n)$ in the Erdös-Renyi random graphs $G(n, p)$. Depending on the choice of $r(n)$ qualitatively different types of behaviour can be observed. We describe the various cases in terms of the quantity $n r^{d}$, which is a measure of the average degree of the graph. Intuitively it should be clear that the expected degree scales with $n r^{d}$. More formally it can be shown that the ratio of the average degree divided by $n r^{d}$ tends to a constant (which depends on $\nu$ ) in probability as long as $n^{2} r^{d} \rightarrow \infty$ (this last condition is needed to ensure that the probability that the graph consists of isolated vertices tends to 0 ).

${ }^{\dagger}$ This author is partially supported by EPSRC, the Department of Statistics, Bekker-la-Bastide fonds, Dr. Hendrik Muller's Vaderlandsch fonds, and Prins Bernhard Cultuurfonds

1365-8050 @ 2005 Discrete Mathematics and Theoretical Computer Science (DMTCS), Nancy, France 


\section{Statement and discussion of results}

Perhaps rather suprisingly at first glance the only feature of the probability measure $\nu$ that plays any role in our results (and proofs) is the maximum density $\nu_{\max }$ defined as: $\sup _{C} \frac{\nu(C)}{\lambda(C)}$, where $\lambda$ denotes the Lebesgue measure on $\mathbb{R}^{d}$ and the supremum is over all measurable sets $C$ with positive Lebesgue measure. We will always assume that $\nu_{\max }<\infty$, which can be seen to be equivalent to $\nu$ having a bounded density function $f$ wrt. the Lebesgue measure.

Theorem 1 The following statements hold, as $n \rightarrow \infty$

(i) If $\frac{n r^{d}}{\ln n} \rightarrow 0$ then $\frac{\chi_{n}}{\omega_{n}} \rightarrow 1$ a.s.

(ii) If $\frac{n r^{d}}{\ln n} \rightarrow \infty$ then $\frac{\chi_{n}}{\omega_{n}} \rightarrow \frac{1}{\delta}$ a.s.

(iii) If $\frac{n r^{d}}{\ln n} \rightarrow t \in(0, \infty)$ then $\frac{\chi_{n}}{\omega_{n}} \rightarrow x(t)$ a.s. Here $1 \leq x(t) \leq \frac{c(t)}{\delta}$,

where $c(t):=\frac{G\left(\left(\frac{\theta}{2^{d} \delta} \nu_{\max } t\right)^{-1}\right)}{G\left(\left(\frac{\theta}{2^{d}} \nu_{\max } t\right)^{-1}\right)}$, and for each $y \geq 0, G(y)$ is the unique $x \geq 1$ that satisfies $x \ln x-x+1=y$.

The constant $\theta$ is the Lebesgue measure $\lambda(B)$ of the unit ball $B:=\{x:\|x\|<1\}$ wrt. $\|$.$\| and \delta$ is the the (translational) packing density of $B$, meaning that $\delta$ is the maximal proportion of $d$-space that can be filled with disjoint translates of $B$. One of several equivalent definitions of $\delta$ is the following. Let $N(K)$ denote the maximum cardinality of mutually disjoint translates of $B$ with centers in $[0, K]^{d}$ then

$$
\delta:=\lim _{K \rightarrow \infty} \frac{\theta N(K)}{K^{d}} .
$$

It can be shown that this limit exists. For an overview of results on packing see for instance (Rog64) or (PA95). In $\mathbb{R}^{2}$ with the Euclidean norm $\delta=\frac{\pi}{2 \sqrt{3}}$, so that $\frac{1}{\delta} \approx 1.103$. Assuming that $\delta \neq 1$ the function $c(t)$ in Theorem 1 is strictly increasing, $c(t) \rightarrow \delta$ as $t \rightarrow 0$ and $c(t) \rightarrow 1$ as $t \rightarrow \infty$. If $\delta=1$ then $c(t) \equiv 1$ and in that case $\lim \frac{\chi}{\omega}=1$ a.s. for all choices of $r(n)$ with $r(n) \rightarrow 0$.

Following the interpretation of $n r^{d}$ as a measure of the average degree, we will refer to the case $\frac{n r^{d}}{\ln n} \rightarrow$ 0 as the sparse case, the case $\frac{n r^{d}}{\ln n} \rightarrow \infty$ as the dense case and the case when $\frac{n r^{d}}{\ln n} \rightarrow t \in(0, \infty)$ as the intermediate case $(s)$. We remark that although the theorem has been phrased in terms of the ratio $\frac{\chi}{\omega}$, the proofs of the results actually describe the behaviour of $\chi$ and $\omega$ separately.

Results similar to Theorem 1 already appeared in (McD03; Pen03). Part (i) of Theorem 1, concerning the sparse case, is in fact a strengthening of a result that appears in both (McD03) and (Pen03), because there the extra condition $n r^{d}=n^{o(1)}$ was assumed, which amounts to discarding the case when the average degree is very small (a negative power of $n$ ), and furthermore both showed convergence in probability as opposed to almost sure convergence. As part of the proof we show that when the average degree is very small then something much stronger than convergence of the ratio to 1 holds, namely: if $n r^{d}=o\left(n^{-\alpha}\right)$ for some $\alpha>0$ then with probability one $\chi_{n}=\omega_{n}$ for all but finitely many $n$.

In the dense case (Pen03) effectively gave an upper bound for lim sup $\frac{\chi}{\omega}$ of $\frac{1}{\delta_{L}}$ and a lower bound for $\lim \inf \frac{\chi}{\omega}$ of $\frac{1}{\delta}$, where $\delta_{L}$ is the so-called lattice packing density of $B$ (ie. the proportion of $d$-space that can be filled with disjoint translates of $B$ whose centers are the integer linear combinations of some basis 
for $\mathbb{R}^{d}$ ). The paper (McD03) only considers the Euclidean norm in the plane in which case $\delta$ and $\delta_{L}$ coincide. In general dimension the question of whether $\delta=\delta_{L}$ is open for the Euclidean norm and it is conjectured that $\delta>\delta_{L}$ for large dimensions $d$ (see $(\operatorname{Rog} 64)$ ).

In the intermediate case our contribution consists of giving an upper bound which is an improvement over the one given in section 6.6 of (Pen03), which in particular shows that $\frac{1}{\delta}$ is always an upper bound; and showing that the (almost sure) limit of $\frac{\chi_{n}}{\omega_{n}}$ exists.

A rough sketch of the proof of the upper bound is as follows. In (Pen03) it was already shown that $\lim \frac{\omega_{n}}{n r^{d}}=\frac{a \theta \nu_{\max }}{2^{d}}$ a.s. where $a=G\left(\left(\frac{\theta}{2^{d}} \nu_{\max } t\right)^{-1}\right)$ in the intermediate case and $a=1$ in the dense case. Thus it remains to show that $\frac{\chi_{n}}{n r^{d}} \leq(1+\epsilon) \frac{b \theta \nu_{\max }}{2^{d} \delta}$ (for all but finitely many $n$, with probability one), where $b=1$ in the dense case and $b=G\left(\left(\frac{\theta}{2^{d} \delta} \nu_{\max } t\right)^{-1}\right)$ in the intermediate case. To do this we dissect $\mathbb{R}^{d}$ into a collection $\mathcal{C}$ of 'small' cubes of side $\eta r$ (with $\eta=\eta(\epsilon)$ small but fixed) and partition $\mathcal{C}$ into subcollections $\mathcal{C}_{1}, \ldots, \mathcal{C}_{N}$ such that $\|p-q\| \geq r$ whenever $p, q$ are in different small cubes belonging to the same $\mathcal{C}_{i}$. Using the definition of $\delta$, the $\mathcal{C}_{i}$ can be constructed in such a way that for any fixed $K>0$ the union of any $N$ small cubes in $\mathcal{C}$ contained in a large cube of side $r K$ will contain no more than $(1+\epsilon) \frac{b \theta \nu_{\max }}{2^{d} \delta} n r^{d}$ points (for all but finitely many $n$, with probability one). Since points in different small cubes belonging to the same $\mathcal{C}_{i}$ may receive the same colours, it follows that we can colour any subgraph of $G_{n}$ induced by the points in a large cube of side $r K$ with no more than $(1+\epsilon) \frac{b \theta \nu_{\max }}{2^{d} \delta} n r^{d}$ colours. These colourings of induced subgraphs can be adapted to yield a colouring of the entire graph with the same number of colours when $K$ is sufficiently large.

To prove the existence result we again consider the chromatic number of subgraphs induced by the points in a cube of side $r K$ for $K$ fixed. When $K$ is large the maximum over all such subgraphs will be a good approximation of the chromatic number of the entire graph and the chromatic number of such a subgraph can in turn be approximated well by a linear program with fixed dimensions (dependent on $K$ and $\epsilon$ if we wish to approximate to within a factor $1+\epsilon$ ). Each of the feasible points $p$ of this linear program corresponds to a sum $\sum_{i=1}^{n} f_{p}\left(X_{i}\right)$ where $f_{p}$ is a particular type of function with range $[0,1]$. The result now follows by considering the probability distribution of such a sum.

In the course of the proof of part (i) we find that when $n r^{d}$ is bounded above by a negative power of $n$ then the probability mass of the clique number becomes concentrated on two consecutive integers in the sense that $\mathbb{P}\left(\omega_{n} \in\{m(n), m(n)+1\}\right) \rightarrow 1$ for some sequence $m(n)$ which depends on $r(n)$. The same holds for the maximum degree and chromatic number. For sparse Erdös-Renyi random graphs a similar phenomenon is well known to occur for several graph parameters including the chromatic number. In (Pen03) it was shown that this phenomenon, dubbed focusing, holds for the maximum degree if $n r^{d}=$ $o(\ln n)$ and it was conjectured to hold for the clique number as well when $n r^{d}=o(\ln n)$. We are able to prove the conjecture and extend the result to the chromatic number:

Theorem 2 If $n r^{d}=o(\ln n)$ then there exist sequences $m(n), k(n)$ such that as $n \rightarrow \infty$

(i) $\mathbb{P}\left(\omega_{n} \in\{m(n), m(n)+1\}\right) \rightarrow 1$;

(ii) $\mathbb{P}\left(\chi_{n} \in\{k(n), k(n)+1\}\right) \rightarrow 1$.

\section{References}

[McD03] C. McDiarmid. Random channel assignment in the plane. Random Structures Algorithms, 22(2):187-212, 2003. 
[PA95] J. Pach and P. K. Agarwal. Combinatorial geometry. Wiley-Interscience Series in Discrete Mathematics and Optimization. John Wiley \& Sons Inc., New York, 1995. A WileyInterscience Publication.

[Pen03] M. D. Penrose. Random Geometric Graphs. Oxford University Press, Oxford, 2003.

[Rog64] C. A. Rogers. Packing and covering. Cambridge Tracts in Mathematics and Mathematical Physics, No. 54. Cambridge University Press, New York, 1964. 